\title{
Preventing Fusion Mass Shift Avoids Postoperative Distal Curve Adding-on in Adolescent Idiopathic Scoliosis
}

\author{
Hideki Shigematsu MD, PhD, \\ Jason Pui Yin Cheung MBBS (HK), MMedSc, FRCS (Edin), FHKCOS, FHKAM (Orth), Mauro Bruzzone MD, \\ Hiroaki Matsumori MD PhD, Kin-Cheung Mak MBBS, FRCSEd, FHKCOS, FHKAM (Orth), Dino Samartzis DSc, \\ Keith Dip Kei Luk MCh (Orth), FRCSE, FRCSG, FRACS, FHKAM (Orth)
}

Received: 25 July 2016/ Accepted: 15 December 2016/Published online: 3 January 2017

(C) The Association of Bone and Joint Surgeons \& 2016

\begin{abstract}
Background Surgery for adolescent idiopathic scoliosis (AIS) is only complete after achieving fusion to maintain the correction obtained intraoperatively. The instrumented or fused segments can be referred to as the "fusion mass". In patients with AIS, the ideal fusion mass strategy has been established based on fulcrum-bending radiographs for main thoracic curves. Ideally, the fusion mass should achieve parallel endplates of the upper and lower instrumented vertebra and correct any "shift" for truncal
\end{abstract}

Each author certifies that he, or a member of his immediate family, has no funding or commercial associations (eg, consultancies, stock ownership, equity interest, patent/licensing arrangements, etc) that might pose a conflict of interest in connection with the submitted article.

All ICMJE Conflict of Interest Forms for authors and Clinical Orthopaedics and Related Research ${ }^{\circledR}$ editors and board members are on file with the publication and can be viewed on request.

Clinical Orthopaedics and Related Research ${ }^{\mathbb{B}}$ neither advocates nor endorses the use of any treatment, drug, or device. Readers are encouraged to always seek additional information, including FDAapproval status, of any drug or device prior to clinical use.

Each author certifies that his institution approved the human protocol for this investigation, that all investigations were conducted in conformity with ethical principles of research, and that informed consent for participation in the study was obtained.

This work was performed at the Department of Orthopaedics and Traumatology, The University of Hong Kong, Hong Kong, China.

\section{H. Shigematsu,}

Jason P. Y. Cheung, M. Bruzzone, H. Matsumori, K.-C. Mak, D. Samartzis, K. D. K. Luk ( $\square)$

Department of Orthopaedics and Traumatology, The University of Hong Kong, Pokfulam, Hong Kong, SAR, China

e-mail: hrmoldk@hku.hk balance. Distal adding-on is an important element to consider in AIS surgery. This phenomenon represents a progressive increase in the number of vertebrae included distally in the primary curvature and it should be avoided as it is associated with unsatisfactory cosmesis and an increased risk of revision surgery. However, it remains unknown whether any fusion mass shift, or shift in the fusion mass or instrumented segments, affects global spinal balance and distal adding-on after curve correction surgery in patients with AIS.

Questions/Purposes (1) To investigate the relationship among postoperative fusion mass shift, global balance, and distal adding-on phenomenon in patients with AIS; and (2) to identify a cutoff value of fusion mass shift that will lead to distal adding-on.

Methods This was a retrospective study of patients with AIS from a single institution. Between 2006 and 2011 we performed 69 selective thoracic fusions for patients with main thoracic AIS. All patients were evaluated preoperatively and at 2 years postoperatively. The Cobb angle between the cranial and caudal endplates of the fusion mass and the coronal shift between them, which was defined as "fusion mass shift", were measured. Patients with a fusion mass Cobb angle greater than $20^{\circ}$ were excluded to specifically determine the effect of fusion mass shift on distal adding-on phenomenon. Fusion mass shift was

\section{H. Shigematsu, H. Matsumori}

Department of Orthopaedic Surgery, Nara Medical University, Nara, Japan

\section{Bruzzone}

Department of Orthopaedics, Hospital Español de Buenos Aires, Buenos Aires, Argentina 
empirically set as $20 \mathrm{~mm}$ for analysis. Therefore, of the 69 patients who underwent selective thoracic fusion, only 52 with a fusion mass Cobb angle of $20^{\circ}$ or less were recruited for study. We defined patients with a fusion mass shift of $20 \mathrm{~mm}$ or less as the balanced group and those with a fusion mass shift greater than $20 \mathrm{~mm}$ as the unbalanced group. A receiver operating characteristic (ROC) curve was used to determine the cutoff point of fusion mass shift for adding-on.

Results Of the 52 patients studied, fusion mass shift ( $>$ $20 \mathrm{~mm}$ ) was noted in $11(21 \%)$, and six of those patients had distal adding-on at final followup. Although global spinal balance did not differ significantly between patients with or without fusion mass shift, the occurrence of adding-on phenomenon was significantly higher in the unbalanced group (55\% (six of 11 patients), odds ratio [OR], 8.6; 95\% CI, 2-39; p < 0.002) than the balanced group (12\% [five of 41 patients]). Based on the ROC curve analysis, a fusion mass shift more than $18 \mathrm{~mm}$ was observed as the cutoff point for distal adding-on phenomenon (area under the curve, 0.70; 95\% CI, 0.5-0.9; likelihood ratio, 5.0; sensitivity, 0.64; specificity, 0.73; positive predictive value, $39 \%$ [seven of 18 patients]; negative predictive value, $88 \%$ [30 of 34 patients]; OR, 4.8; 95\% CI, 1-20; p = 0.02).

Conclusions Our study illustrates the substantial utility of the fulcrum-bending radiograph in determining fusion levels that can avoid fusion mass shift; thereby, underlining its importance in designing personalized surgical strategies for patients with scoliosis. Preoperatively, determining fusion levels by fulcrum-bending radiographs to avoid residual fusion mass shift is imperative. Intraoperatively, any fusion mass shift should be corrected to avoid distal adding-on, reoperation, and elevated healthcare costs.

Level of Evidence Level II, prognostic study.

\section{Introduction}

The aim of surgical treatment of adolescent idiopathic scoliosis (AIS) is to achieve a balanced spine in the coronal and sagittal planes without listing (deviation in C7-center sacral vertical line) or truncal shifting $[16,30,42]$. In the selection of fusion levels, an ideal fusion mass requires parallel top and bottom endplates without substantial coronal shift, and appropriate sagittal alignment [30, 42]. As proposed by Harrington [13, 14], a simple way to achieve a parallel fusion mass is to instrument from a horizontal vertebrae above the curve to a horizontal vertebrae below the curve. Based on a standing posteroanterior (PA) plain radiograph, the lower end of the fusion should be in the "stable zone" [14]. Goldstein [10, 11], Risser [41], and Moe [36] have advocated fusion from the upper to the lower neutral vertebrae. However, with these criteria, one or two levels beyond the primary curve at both ends are frequently included during surgery. With the development of modern pedicle screw instrumentation systems [18, 27, 45, 54], better 3-dimensional deformity correction with a shorter fusion mass can be achieved [42].

During the last decade, the Lenke et al. [22] classification of AIS was proposed in an attempt to develop new guidelines for fusion-level selection. However, some studies were retrospective, based predominantly on specific curve types, and did not account for curve flexibility [7, 21, 46]. Variable methods of evaluating curve flexibility exist such as traction [51], supine side bending [19], push prone [20], and fulcrum-bending radiographs [2-5, 23-26, 29, 30, 32, 42, 47]. Fulcrum-bending radiographs are performed by placing a fulcrum, a padded and radiolucent cylinder, under the rib corresponding to the apex of the curve with the patient placed in the lateral decubitus position $[2,3]$. This method uses passive rather than active forces and avoids ionizing radiation exposure to the healthcare practitioner. The fulcrum-bending radiograph has been used as a clinical decision tool to determine selection of fusion levels and in predicting curve correction [2-5, 12, 15, 23-26, 28-30, 32, 38, 42, 47]. Essentially, fulcrum-bending radiographs help determine the shortest fusion segment that can achieve a fusion mass with parallel endplates and no shift in an effort to obtain a balanced spine [30, 42]. There are two components of a fusion mass that determine the success or failure of the surgery, namely residual shift and Cobb angle [30]. For purposes of this study and discussion, our definition for a balanced fusion mass is Cobb $20^{\circ}$ or less and shift $20 \mathrm{~mm}$ or less.

Having a fusion mass that is short is important because preserving more mobile spinal segments may help prevent early adjacent segment degeneration [31, 33, 37]. However, surgeons must not fuse short to preserve mobility without adhering to the ultimate goal of balancing the spine. If the fusion mass is too short and does not have parallel upper and lower endplates, the result may be an "adding-on" phenomenon and spinal decompensation $[30,44]$. Distal adding-on is an important element to consider in AIS surgery. This phenomenon represents a progressive increase in the number of vertebrae included distally in the primary curvature combined with an increase of more than $5 \mathrm{~mm}$ deviating from the center sacral vertical line in the first vertebrae caudal to the lower instrumented vertebrae or an increase of more than $5^{\circ}$ in angulation of the first disc caudal to the lower instrumented vertebrae. Adding-on should be avoided as it is associated with unsatisfactory cosmesis and an increased risk of revision surgery [44, 48]. The cause of adding-on is multifactorial, including the selection of the 
lower instrumented vertebrae [53], rotation of the lumbar vertebra [39], and postoperative shoulder imbalance [1]. The effect of an inadequately squared fusion mass on residual truncal shift and coronal imbalance leading to adding-on is unknown.

We believe that a residual shift in the fusion mass or endplates that are not parallel results in decompensation by recruitment of unfused segments above and below by wedging vertebrae and discs to obtain coronal balance. We also think that postoperative shoulder imbalance may be a compensatory mechanism for a shift in the fusion mass. As such, we aimed to clarify the effect of the fusion mass with residual shift or rather the fusion mass shift on (1) the global spinal balance and (2) adding-on phenomenon. An additional objective was to test the accuracy of our previously arbitrarily defined $20-\mathrm{mm}$ cutoff value [30] as an acceptable fusion mass shift.

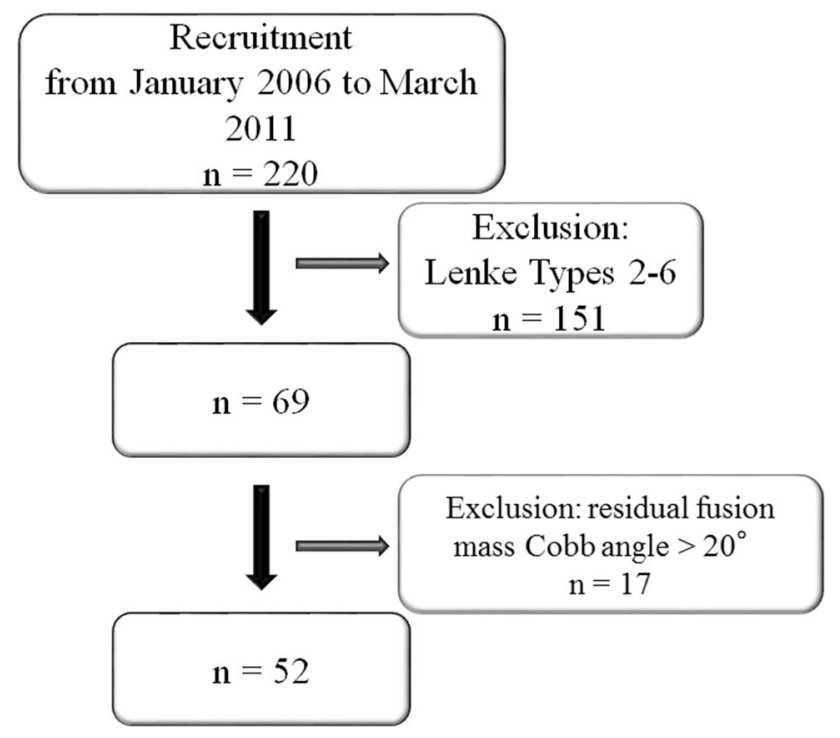

Fig. 1 A flowchart shows the recruitment process of patients in this study.

\section{Patients and Methods}

This was a retrospective study of patients with AIS from a single institution. The study received ethics approval by the local institutional review board. Between 2006 and 2011 we performed 69 selective thoracic fusions for patients with main thoracic AIS (Lenke Types 1A, 1B, or 1C), with alternate level pedicle-screw fixation. Of those, 17 patients were excluded owing to a fusion mass Cobb angle greater than $20^{\circ}$, which left 52 patients for analysis (Fig. 1). The numbers of patients in each group, according to fusion mass shift, were as follows: Balanced group: 41, Unbalanced group: 11 . The fusion mass Cobb angle values were similar in both groups (Table 1). All patients (100\%) had minimum 2 years postoperative followup. Demographic (Table 2) and radiographic (Table 3) data preoperatively and 2 years postoperatively (Table 4) did not differ between the two groups, except for the distal adding-on phenomenon. Patients with neuromuscular scoliosis and congenital scoliosis were excluded. All patients were allowed free mobilization after surgery. Despite possible interplay between fusion mass Cobb angle and fusion mass shift, since the aim of the study was to assess the effect of fusion mass shift, patients with a postoperative fusion mass Cobb angle greater than $20^{\circ}$ were excluded to focus the analysis on the pure effect of residual fusion mass shift on balance and adding-on.

An ideal fusion mass should achieve a balanced spine with the cranial and the caudal endplates of the fusion mass being parallel in the coronal plane without substantial shift and a normal sagittal alignment. Furthermore, the shortest fusion levels were determined that fulfilled the balance criterion while preserving the most number of mobile segments [30].

Based on the fulcrum-bending radiograph, the fusion levels were chosen following the strategy described by Luk et al. [30] in which we aimed to achieve a fusion mass with a fusion mass Cobb angle $20^{\circ}$ or less and fusion mass shift $20 \mathrm{~mm}$ or less. We describe the steps that addressed our strategy as follows (Fig. 2): (1) A line was drawn parallel to the inferior endplate of the estimated lower instrumented vertebrae on the fulcrum-bending radiograph; (2) a line

Table 1. Fusion mass shape in terms of fusion mass Cobb angle and fusion mass shift

\begin{tabular}{|c|c|c|c|c|c|}
\hline \multirow[t]{2}{*}{ Unbalanced fusion mass } & \multirow[t]{2}{*}{ Group } & \multirow[t]{2}{*}{ Mean } & \multirow[t]{2}{*}{ SD } & \multicolumn{2}{|c|}{$95 \% \mathrm{CI}$} \\
\hline & & & & Lower & Upper \\
\hline \multirow[t]{2}{*}{ Fusion mass Cobb angle $\left(^{\circ}\right)$} & Balanced & 13 & 4 & 12 & 14 \\
\hline & Unbalanced & 12 & 6 & 9 & 16 \\
\hline \multirow[t]{2}{*}{ Fusion mass shift (mm) } & Balanced & 11 & 6 & 0 & 20 \\
\hline & Unbalanced & 25 & 3 & 21 & 30 \\
\hline
\end{tabular}

Balanced group = fusion mass shift $20 \mathrm{~mm}$ or less; Unbalanced group = fusion mass shift greater than $20 \mathrm{~mm}$. 
Table 2. Comparison of radiographic parameters

\begin{tabular}{|c|c|c|c|}
\hline Radiographic parameters & $\begin{array}{l}\text { Balanced group } \\
\text { (fusion mass shift } \leq 20 \mathrm{~mm} \text { ) } \\
\text { Mean } \pm \mathrm{SD}\end{array}$ & $\begin{array}{l}\text { Unbalanced group } \\
\text { (fusion mass shift }>20 \mathrm{~mm} \text { ) } \\
\text { Mean } \pm \mathrm{SD}\end{array}$ & $\mathrm{p}$ Value \\
\hline \multicolumn{4}{|l|}{ Preoperative } \\
\hline Proximal thoracic curve $\left(^{\circ}\right)$ & $30 \pm 7$ & $32 \pm 6$ & 0.92 \\
\hline Main thoracic curve $\left(^{\circ}\right)$ & $57 \pm 11$ & $56 \pm 11$ & 1.0 \\
\hline Main thoracic curve flexibility & $61 \pm 16$ & $67 \pm 19$ & 0.76 \\
\hline Lumbar curve $\left(^{\circ}\right)$ & $33 \pm 8$ & $34 \pm 11$ & 0.94 \\
\hline Trunk shift $(\mathrm{mm})$ & $22 \pm 14$ & $24 \pm 12$ & 0.99 \\
\hline List (mm) & $3 \pm 13$ & $0.3 \pm 14$ & 0.95 \\
\hline Radiographic shoulder height (mm) & $-0.1 \pm 8$ & $-2 \pm 8$ & 0.94 \\
\hline T1 tilt $\left({ }^{\circ}\right)$ & $0.4 \pm 5$ & $-0.3 \pm 5$ & 0.98 \\
\hline T5-T12 kyphosis $\left({ }^{\circ}\right)$ & $18 \pm 14$ & $16 \pm 11$ & 0.98 \\
\hline T12-S1 lordosis $\left({ }^{\circ}\right)$ & $53 \pm 12$ & $48 \pm 11$ & 0.66 \\
\hline Sagittal balance (mm) & $-17 \pm 25$ & $-15 \pm 20$ & 0.99 \\
\hline \multicolumn{4}{|l|}{ Postoperative 2-year followup } \\
\hline Proximal thoracic curve $\left(^{\circ}\right)$ & $16 \pm 7$ & $15 \pm 5$ & 0.97 \\
\hline Proximal thoracic correction rate $(\%)$ & $48 \pm 18$ & $52 \pm 14$ & 0.87 \\
\hline Main thoracic curve $\left(^{\circ}\right)$ & $16 \pm 5$ & $17 \pm 5$ & 0.93 \\
\hline Main thoracic curve correction rate $(\%)$ & $71 \pm 9$ & $68 \pm 11$ & 0.77 \\
\hline Lumbar curve $\left(^{\circ}\right)$ & $10 \pm 7$ & $12 \pm 10$ & 0.84 \\
\hline Lumbar correction rate $(\%)$ & $72 \pm 19$ & $65 \pm 22$ & 0.80 \\
\hline Trunk shift (mm) & $-5 \pm 11$ & $-2 \pm 12$ & 0.84 \\
\hline List (mm) & $-6 \pm 12$ & $-3 \pm 18$ & 0.88 \\
\hline Radiographic shoulder height (mm) & $-9 \pm 7$ & $-11 \pm 10$ & 0.95 \\
\hline T1 tilt $\left(^{\circ}\right)$ & $6 \pm 5$ & $4 \pm 6$ & 0.91 \\
\hline T5-T12 kyphosis $\left({ }^{\circ}\right)$ & $21 \pm 8$ & $14 \pm 6$ & 0.09 \\
\hline T12-S1 lordosis $\left({ }^{\circ}\right)$ & $57 \pm 10$ & $54 \pm 17$ & 0.92 \\
\hline Sagittal balance (mm) & $-23 \pm 29$ & $-11 \pm 27$ & 0.60 \\
\hline
\end{tabular}

perpendicular to the above line was drawn from the center of the lower instrumented vertebrae; this was termed the central line; (3) a line parallel to the superior endplate of the intended upper instrumented vertebrae was drawn; (4) the shortest segments that met the following criteria were chosen as the instrumented fusion levels: (a) fusion mass Cobb angle $20^{\circ}$ or less (between the intended lower instrumented vertebrae and upper instrumented vertebrae); and (b) a shift of the upper instrumented vertebrae by 20 $\mathrm{mm}$ or less on either side of the central line (fusion mass shift); (5) if the upper instrumented vertebrae shift was greater than $20 \mathrm{~mm}$ from the central line or fusion mass Cobb angle greater than $20^{\circ}$ (between the lower instrumented vertebrae and upper instrumented vertebrae), the next caudal vertebra was used as the lower instrumented vertebrae, and steps 1 to 4 were repeated; (6) adding levels cranially improved the fusion mass Cobb angle only, whereas adding levels caudally improved the fusion mass shift and the fusion mass Cobb angle [30].
Intraoperatively, radiographs were taken after instrumentation to confirm the fusion mass appearance. Any inadequate fusion mass appearance was further corrected with compression or distraction forces at the lower instrumented vertebrae (Fig. 3).

All image data were available in our archiving and communication systems. Standing PA and lateral digital radiographs were reviewed preoperatively, immediately postoperatively, and 2 years after surgery. Furthermore, we measured the 2-year postoperative standing radiographs for analysis of fusion mass shift and distal adding-on phenomenon. All images were digitized and imported to a DICOM-based computer software program (Radworks ${ }^{\mathrm{TM}}$ 5.1; Applicare Medical Imaging BV, Zeist, The Netherlands). Two readers (HS, HM), not involved in the care of these patients, performed the measurements independently and blinded to the patient information. Preoperative and postoperative images were measured with random allocation to avoid bias in relation to the patient identity and outcome. A 
Table 3. Additional demographic and radiographic parameters

\begin{tabular}{|c|c|c|c|}
\hline Categorical factors & $\begin{array}{l}\text { Balanced group } \\
\text { (fusion mass shift } \leq 20 \mathrm{~mm} \text { ) }\end{array}$ & $\begin{array}{l}\text { Unbalanced group } \\
\text { (fusion mass shift }>20 \mathrm{~mm} \text { ) }\end{array}$ & $\mathrm{p}$ Value \\
\hline \multicolumn{4}{|l|}{ Preoperative } \\
\hline \multicolumn{4}{|l|}{ Sex } \\
\hline Male (number) & 3 & 3 & \\
\hline Female (number) & 38 & 8 & 0.066 \\
\hline \multicolumn{4}{|l|}{ Risser grade } \\
\hline $0(\mathrm{n})$ & 3 (closed triradiate cartilage) & 1 (closed triradiate cartilage) & \\
\hline $1(\mathrm{n})$ & 4 & 1 & \\
\hline $2(\mathrm{n})$ & 4 & 1 & \\
\hline $3(n)$ & 1 & 1 & \\
\hline $4(n)$ & 21 & 6 & \\
\hline $5(\mathrm{n})$ & 8 & 1 & 0.90 \\
\hline \multicolumn{4}{|c|}{ Lenke Type 1 modifier } \\
\hline $\mathrm{A}(\mathrm{n})$ & 28 & 6 & \\
\hline $\mathrm{B}(\mathrm{n})$ & 10 & 2 & \\
\hline $\mathrm{C}(\mathrm{n})$ & 3 & 3 & 0.18 \\
\hline \multicolumn{4}{|l|}{ Truncal shift } \\
\hline Balanced (n) & 15 & 4 & \\
\hline Imbalanced (n) & 25 & 7 & 0.95 \\
\hline \multicolumn{4}{|l|}{ Listing } \\
\hline Balanced (n) & 31 & 8 & \\
\hline Imbalanced (n) & 10 & 3 & 0.85 \\
\hline \multicolumn{4}{|c|}{ Radiographic shoulder height } \\
\hline Balanced (n) & 34 & 9 & \\
\hline Imbalanced (n) & 7 & 2 & 0.93 \\
\hline
\end{tabular}

separate investigator (JPYC) from the two readers randomly chose 20 cases for interobserver reliability analysis of preoperative and postoperative measurements.

Fusion mass shift was the magnitude of the upper instrumented vertebrae shift from the lower instrumented vertebrae. This was measured on the 2-year postoperative standing PA radiographs. First, a line was drawn parallel to the inferior endplate of the lower instrumented vertebrae. Next, a line perpendicular to this line was drawn from the center of the lower instrumented vertebrae to the central line. The distance from the central line to the midpoint of the upper endplate of the upper instrumented vertebrae then was measured as fusion mass shift (Fig. 4). Patients were divided into two groups according to the empirical cutoff of $20 \mathrm{~mm}$ for fusion mass shift: balanced group = fusion mass shift $20 \mathrm{~mm}$ or less, unbalanced group = fusion mass shift greater than $20 \mathrm{~mm}$.

On each standing PA radiograph, the center sacral vertical line, the vertical line bisecting the proximal sacrum, was first drawn followed by measurement of the translation (deviation from the center sacral vertical line) in terms of listing and truncal shift. The radiographic shoulder height and T1 tilt also were measured to evaluate shoulder balance
[30]. Right side up was considered positive for radiographic shoulder height, and left side up was considered positive for T1 tilt. For sagittal alignment evaluation, we measured thoracic kyphosis (T5-T12), lumbar lordosis (T12-S1), and the distance from the C7 plumb line.

Radiographic shoulder height was defined as the side-toside difference in height of the intersection points between the upper surface of the clavicle and a vertical line drawn at $10 \mathrm{~cm}$ from the midline division of the medial ends of the clavicles (Fig. 5) [30]. Radiographic shoulder height was determined based on the standing PA radiograph. A difference greater than $10 \mathrm{~mm}$ was defined as imbalance [43].

Listing was measured by the deviation from a vertical line drawn from the $\mathrm{C} 7$ spinous process to the $\mathrm{S} 1$ spinous process [9, 17, 30, 35, 40] (Fig. 6). A listing greater than 20 $\mathrm{mm}$ was defined as imbalance.

Truncal shift in the coronal plane was determined by first identifying the apical thoracic vertebrae [8, 30, 49]. Through the centroid, a horizontal reference line was drawn. Two points were marked at the intersection of the horizontal reference line and the rib cage on the left and right, at the apical thoracic vertebrae level. The midpoint of this line was marked. A perpendicular line from this 
Table 4. Postoperative radiographic parameters at 2-year followup

\begin{tabular}{|c|c|c|c|}
\hline Categorical factors & $\begin{array}{l}\text { Balanced group } \\
\text { (fusion mass shift } \leq 20 \mathrm{~mm} \text { ) }\end{array}$ & $\begin{array}{l}\text { Unbalanced group } \\
\text { (fusion mass shift }>20 \mathrm{~mm} \text { ) }\end{array}$ & $\mathrm{p}$ Value \\
\hline \multicolumn{4}{|c|}{ Upper instrumented vertebra level } \\
\hline T3 or $4(n)$ & 5 & 1 & \\
\hline T5 or $6(n)$ & 36 & 10 & \\
\hline $\mathrm{T} 7(\mathrm{n})$ & 0 & 0 & 0.78 \\
\hline \multicolumn{4}{|c|}{ Lower instrumented vertebra level } \\
\hline T11 or T12 (n) & 20 & 5 & \\
\hline L1 or L2 (n) & 21 & 6 & 0.85 \\
\hline \multicolumn{4}{|l|}{ Truncal shift } \\
\hline Balanced (n) & 36 & 8 & \\
\hline Imbalanced (n) & 5 & 3 & 0.22 \\
\hline \multicolumn{4}{|l|}{ Listing } \\
\hline Balanced (n) & 33 & 6 & \\
\hline Imbalanced (n) & 8 & 5 & 0.080 \\
\hline \multicolumn{4}{|c|}{ Radiographic shoulder height } \\
\hline Balanced (n) & 21 & 5 & \\
\hline Imbalanced (n) & 20 & 6 & 0.73 \\
\hline \multicolumn{4}{|c|}{ Adding-on phenomenon } \\
\hline Presence (n) & 5 & 6 & \\
\hline Absence (n) & 36 & 5 & $0.002^{*}$ \\
\hline
\end{tabular}

Statistically significant difference.

midpoint was used as a reference line. The perpendicular distance from the central sacral line to a line that bisected the distance from the lateral edges of the rib (Fig. 6) was measured. A truncal shift greater than $20 \mathrm{~mm}$ was defined as imbalance.

Distal adding-on phenomenon, described by Wang et al. [53], was measured comparing the 2-year postoperative standing PA radiographs with the immediate postoperative standing PA radiographs. It was defined as a progressive increase in the number of vertebra included in the main curvature combined with an increase greater than $5 \mathrm{~mm}$ in the deviation of the first vertebra below the lower instrumented vertebrae or an increase greater than $5^{\circ}$ in the angulation of the first disc below the lower instrumented vertebrae. We determined that the disc angle open to the right was positive and that open to the left was negative. In addition, if the lower instrumented vertebrae plus one centroid was on the right side of the center sacral vertical line, the distance was positive.

Regarding key radiographic parameters, interobserver reliability was found to be good to excellent for assessment of the preoperative main thoracic Cobb angle $(\alpha=0.97$; 95\% CI, 0.93-0.99; $\mathrm{p}<0.001)$, the preoperative fusion mass Cobb angle ( $\alpha=0.98 ; 95 \%$ CI, 0.94-0.99; $p<0.001$ ), T1 tilt $(\alpha=0.94 ; 95 \% \mathrm{CI}, 0.86-0.98 ; \mathrm{p}<0.001)$, listing $(\alpha=0.97 ; 95 \%$ CI, 0.92-0.99; p $<0.001)$, truncal shift ( $\alpha=0.98 ; 95 \%$ CI, 0.96-0.99; p $<0.001)$, postoperative
Cobb angle ( $\alpha=0.93 ; 95 \%$ CI, 0.82-0.97; $p<0.001$ ), and fusion mass shift ( $\alpha=0.97$; 95\% CI, 0.93-0.99; $\mathrm{p}<0.001$ ).

\section{Statistical Analysis}

All data were collected and statistical analyses were performed with SPSS, Version 17.0 (SPSS Inc, Chicago, IL, USA). The measurement data including radiographic shoulder height, listing, and truncal shift, were converted to a categorical scale as balanced or imbalanced based on the definitions described above. To determine whether our grouping cutoff of fusion mass shift greater than $20 \mathrm{~mm}$ was appropriate, the receiver operating characteristic (ROC) of the curve analysis was used to assess the best cutoff value regarding fusion mass shift that would lead to postoperative distal adding-on phenomenon. Area under the curve (AUC) analysis was performed and the best sensitivity and specificity results were selected to represent the cutoff value, which was compared with our previously reported $20 \mathrm{~mm}$ shift value. The ideal sensitivity and specificity cutoff values were determined by the corresponding reference line point that closely corresponded to the AUC value of 1 . After the ROC analysis, the cutoff value was selected and based on that, odds ratio analysis was performed to address the development of the distal adding-on phenomenon. Cronbach's intraclass coefficient testing was used to assess 

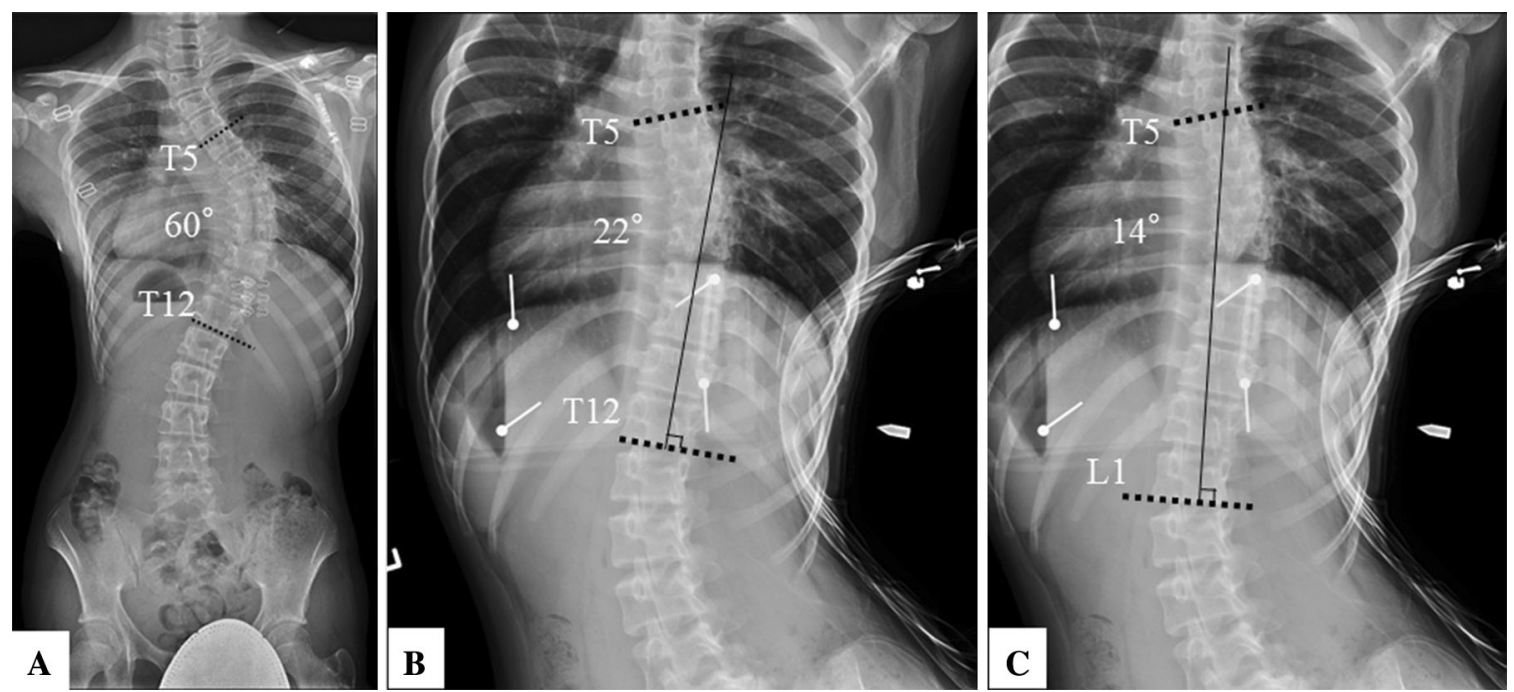

Fig. 2A-C The technique used for determining the ideal fusion level based on fulcrum bending radiographs is shown. (A) A representative patient with adolescent idiopathic scoliosis has a Cobb angle of $60^{\circ}$ from T5 to T12. (B) Based on the fulcrum-bending radiograph, if we fuse from $\mathrm{T} 5$ to $\mathrm{T} 12$, the residual fusion mass Cobb angle will be $22^{\circ}$ but with a shift greater than $20 \mathrm{~mm}$. (C) If we fuse down to L1, the fusion mass Cobb angle will become $14^{\circ}$ with an expected fusion mass shift less than $20 \mathrm{~mm}$.
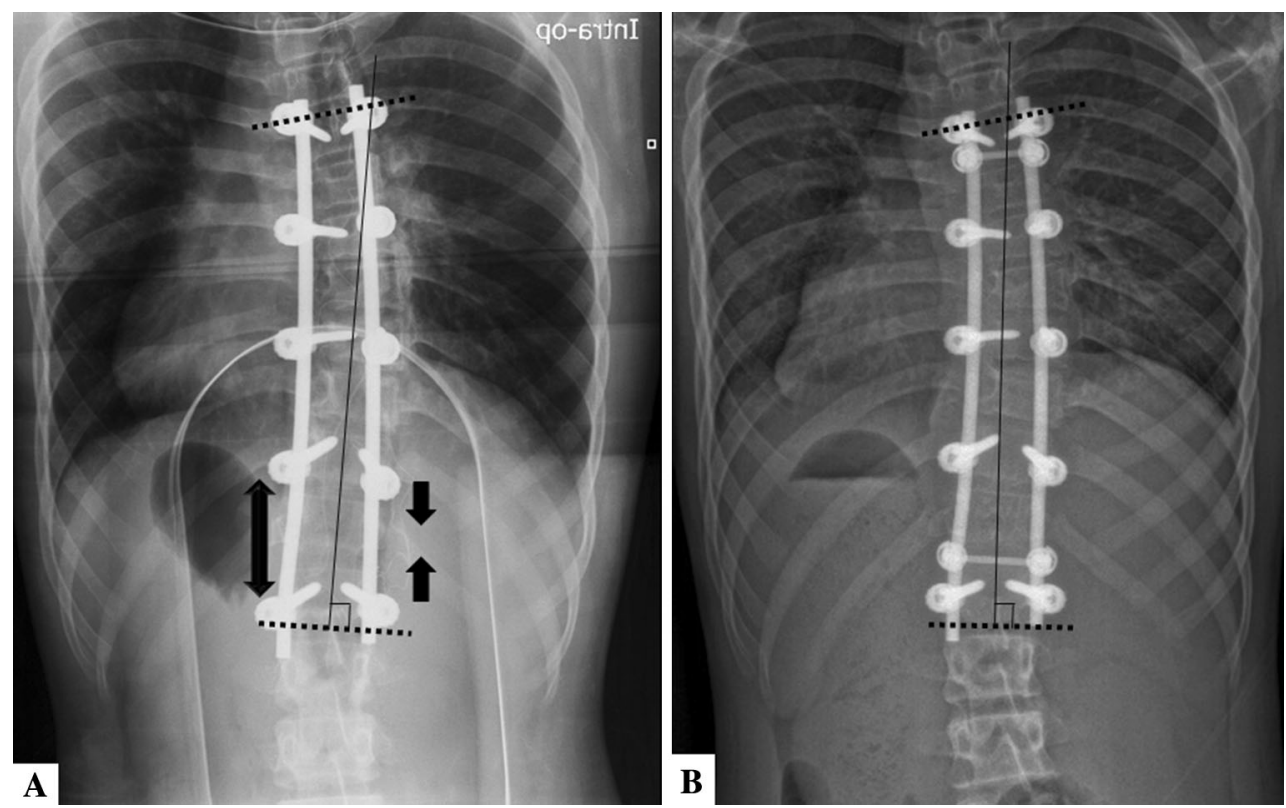

Fig. 3A-B (A) A patient's intraoperative radiograph after preliminary instrumentation shows inadequate correction of the fusion mass shift. Therefore, further distraction on the concave side and further

reliability of the measurements, whereby $\alpha$ values were obtained. The strength of reliability assessments was regarded as follows: $\alpha$ values of 0.90 or greater were excellent, 0.80 or greater were good, 0.70 or greater fair, and less than 0.70 were poor [50]. Only good to excellent reliability was accepted. Any differences were settled by consensus. The significance level was defined as a probability less than $0.05 .95 \%$ CIs were noted to assess precision. compression of the convex side of the curve were performed. (B) An immediate postoperative radiograph shows satisfactory correction of the fusion mass shift.

\section{Results}

Patients with fusion mass shift $20 \mathrm{~mm}$ or less were less likely to have adding-on at 2-year followup than were patients with fusion mass shift greater than $20 \mathrm{~mm}(12 \%$ [five of 41 patients] versus $55 \%$ [six of 11 patients]; odds ratio $[\mathrm{OR}], 8.6 ; 95 \% \mathrm{CI}, 2-39 ; \mathrm{p}=0.002$ ) (Fig. 7). There were no differences between the groups for factors related 


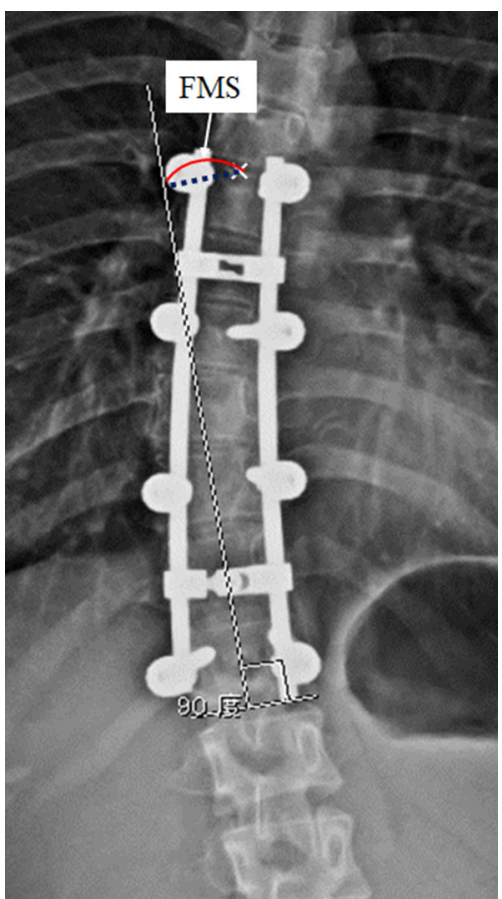

Fig. 4 The measurement guide for fusion mass shift is shown. The distance between the midpoint of the upper vertebral border and a line drawn perpendicular to the lower vertebral border is defined as the fusion mass shift (FMS)

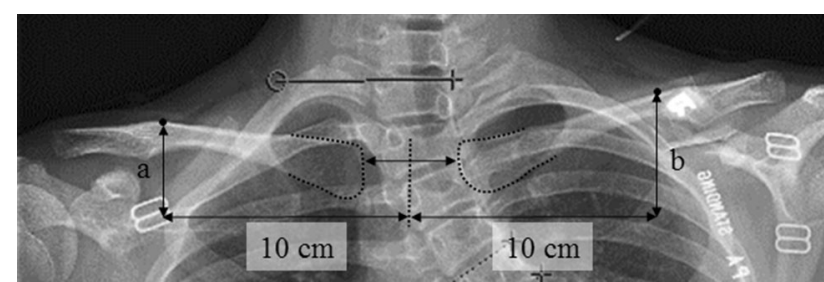

Fig. 5 A detailed measurement of radiographic shoulder height by calculating the difference between points $\mathrm{a}$ and $\mathrm{b}(\mathrm{mm})$ is shown.

to distal adding-on including the lower instrumented vertebrae, lower instrumented vertebrae plus one disc angle, and lower instrumented vertebrae plus one shift at immediate or 2-year postoperative assessment (Table 5). The ROC curve was examined to determine the cutoff point of fusion mass shift that would cause a distal adding-on phenomenon. A fusion mass shift more than $18 \mathrm{~mm}$ was observed as the cutoff point that best identified the likelihood of a distal adding-on phenomenon (AUC, 0.70; 95\% CI, 0.5-0.9; sensitivity, 0.64, specificity, 0.73; $\mathrm{p}<0.05$ ). Patients with a residual fusion mass shift greater than 18 $\mathrm{mm}$ had an increased risk of having a distal adding-on phenomenon (likelihood ratio, 5.0; positive predictive value, $39 \%$ [seven of 18 patients]; negative predictive value, $88 \%$ [30 of 34 patients]; OR, $4.8 ; 95 \% \mathrm{CI}, 1-20 ; \mathrm{p}=$ 0.02) (Table 6). When the cutoff point of the fusion mass

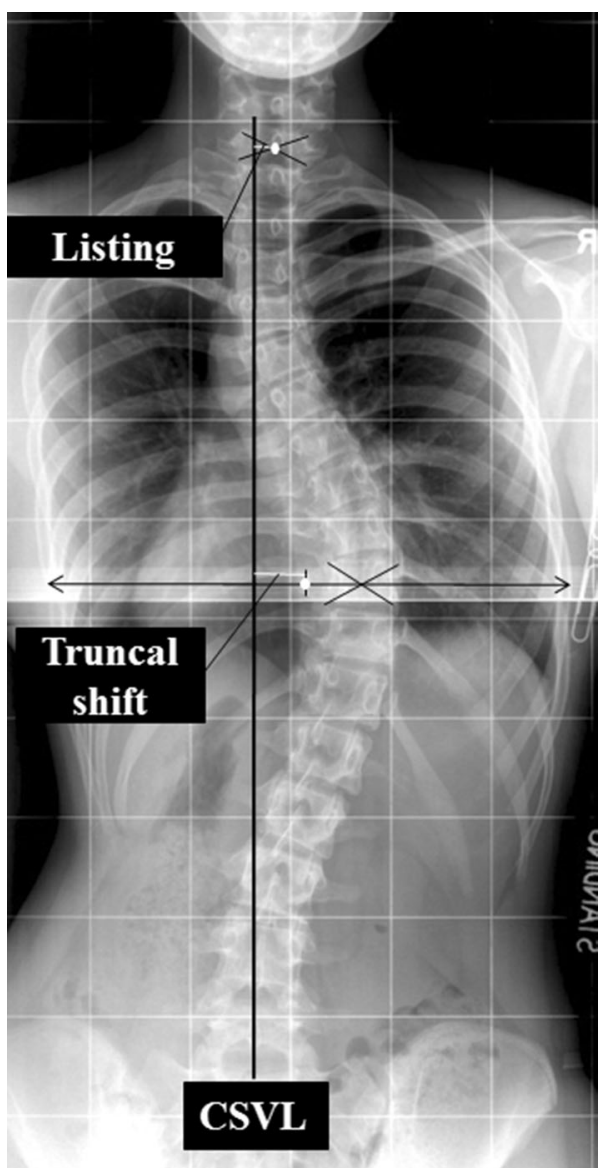

Fig. 6 A measurement guide of truncal shift and list is shown. Truncal shift is measured by plotting the central sacral vertical line (CSVL) and measuring the deviation of the midpoint of the two widest points of the rib cage along a horizontal parallel line. Listing is measured by plotting the $\mathrm{C} 7$ plumb line and measuring the deviation between the mid-point of the intercrestal line.

shift was $20 \mathrm{~mm}$, which was the original published criterion [30], the sensitivity was 0.55 and the specificity was 0.85 . None of our patients underwent additional surgery to treat the adding-on phenomenon.

\section{Discussion}

Primarily, our surgical strategy for managing AIS involves an easy and logical method to understand what the ideal extent of fusion should be, based on preoperative fulcrumbending radiographs. The acceptable fusion mass is defined as fusion mass shift $20 \mathrm{~mm}$ or less and fusion mass Cobb angle $20^{\circ}$ or less [30]. These criteria help to avoid residual deformities that may require other unfused segments of the spine to compensate. This is consistent with the goals of AIS surgery in which the deformity can be corrected while rebalancing the global spine. Despite the possible interplay between fusion mass shift and fusion mass Cobb angle, for 

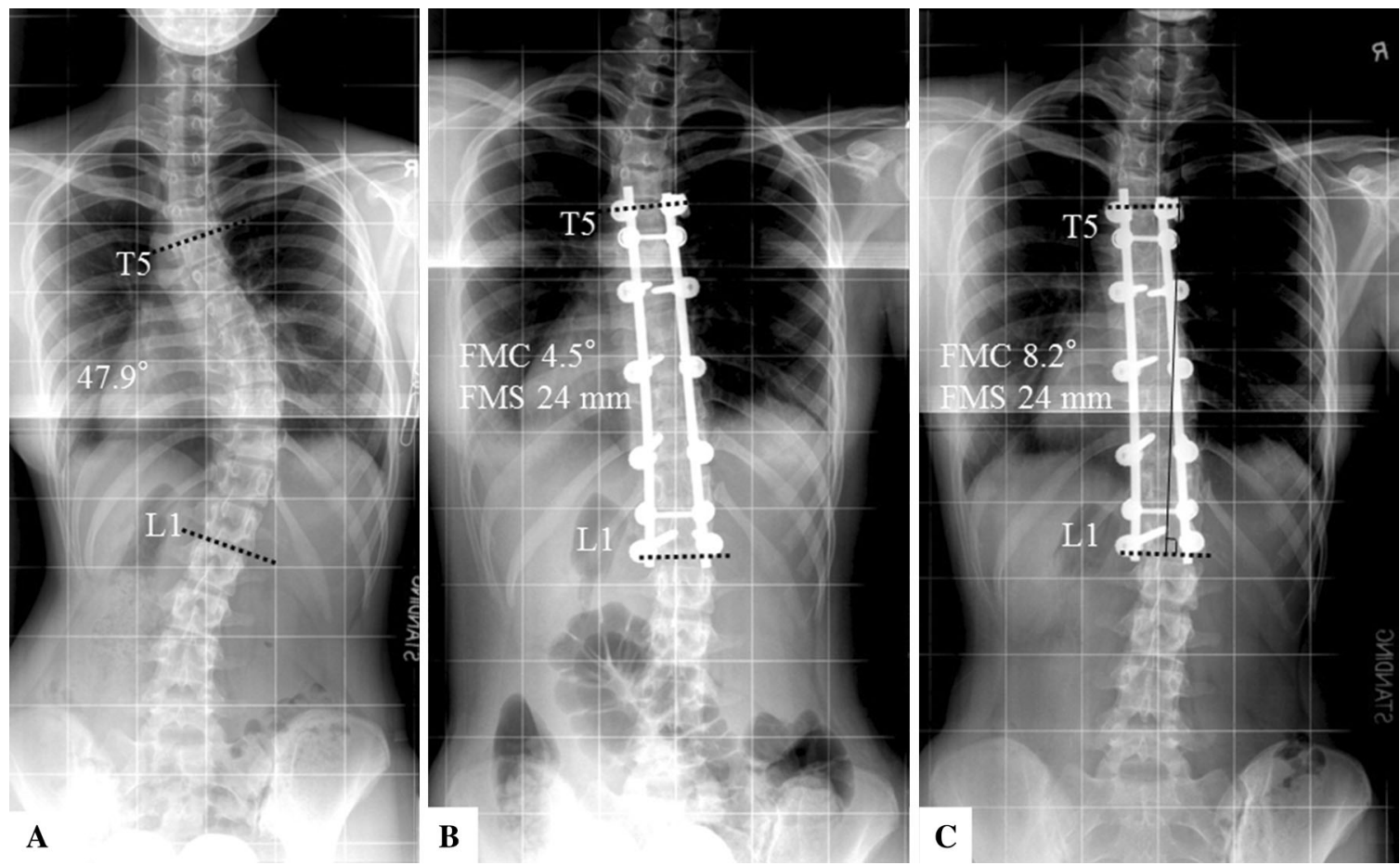

Fig. 7A-C A 14-year-old girl had a residual fusion mass shift (FMS) of $24 \mathrm{~mm}$ with postoperative adding-on phenomenon developing despite good correction of the fusion mass Cobb (FMC) angle. (A) The Cobb angle was $48^{\circ}$ from T5 to L1 before surgery. (B)
Immediately postoperative, the fusion mass Cobb angle was $4.5^{\circ}$. (C) At the 2-year followup, the fusion mass Cobb angle was $8^{\circ}$ with distal adding-on phenomenon.

Table 5. Factors of distal adding-on phenomenon

\begin{tabular}{lll}
\hline Radiographic parameters & $\begin{array}{l}\text { Balanced group } \\
\text { (fusion mass shift } \leq 20 \mathrm{~mm})\end{array}$ & $\begin{array}{l}\text { Unbalanced group } \\
\text { (fusion mass shift }>20 \mathrm{~mm} \text { ) }\end{array}$ \\
\hline $\begin{array}{l}\text { Immediate postoperative } \\
\begin{array}{l}\text { Lower instrumented vertebrae/lower instrumented } \\
\text { vertebrae }+1 \text { disc angle }\end{array}\end{array}$ & $-0.1 \pm 3$ & $-2 \pm 6$ \\
$\begin{array}{l}\text { Lower instrumented vertebrae }+1 \text { shift } \\
\begin{array}{l}\text { Postoperative 2-year followup } \\
\text { Lower instrumented vertebrae/lower instrumented } \\
\text { vertebrae }+1 \text { disc angle }\end{array}\end{array}$ & $-12 \pm 12$ & $-13 \pm 16$ \\
$\quad$ Lower instrumented vertebrae +1 shift & $-8 \pm 12$ & $0.5 \pm 6$ \\
\hline
\end{tabular}

Values $=$ mean \pm SD.

the purposes of this study, we focused on fusion mass shift at a standardized fusion mass Cobb angle to determine the pure effect of residual fusion mass shift on overall spinal balance and adding-on. In this study, a distal adding-on phenomenon was observed in $21 \%$ ( 11 of 52 patients) of all patients at the 2-year followup, comparable to a rate of $18.8 \%$ reported previously for Lenke Type 1A curves [34]. Other authors have reported an incidence of distal adding-on from $21 \%$ to $51.1 \%$ [6, 53]. Adding-on has been observed in patients, especially those with remaining growth potential, with residual shoulder imbalance [1], inappropriate lower instrumented vertebrae [6, 52, 53], and residual apical translation [34]. However, to our knowledge, whether the fusion mass shift can cause distal adding-on has not been reported. To our knowledge, our study is the first to report on the concept of fusion mass shift and its effects on the distal adding-on phenomenon. In our patients, the distal adding-on phenomenon was reduced to $12 \%$ (five of 41 patients) when we achieved a balanced fusion mass with a fusion mass shift of $20 \mathrm{~mm}$ or less and a fusion mass Cobb angle of $20^{\circ}$ or less. 
Table 6. Postoperative radiographic parameters with fusion mass shift criteria as $18 \mathrm{~mm}$

\begin{tabular}{lll}
\hline Categorical factors & $\begin{array}{l}\text { Balanced group } \\
\text { Fusion mass shift } \leq 18 \mathrm{~mm}, \\
\text { fusion mass Cobb angle } \leq 20^{\circ}\end{array}$ & $\begin{array}{l}\text { Unbalanced group } \\
\text { Fusion mass shift }>18 \mathrm{~mm}, \\
\text { fusion mass Cobb angle } \leq 20^{\circ}\end{array}$ \\
\hline $\begin{array}{l}\text { Adding-on at postoperative final followup } \\
\text { Positive }\end{array}$ & 11 & 7 \\
Negative & 30 & 4 \\
\hline
\end{tabular}

* Statistically significant.

This study has several limitations. First, the sample size is relatively small because of the single-institution study design but it is one of the largest studies addressing patients with AIS and the fulcrum-bending radiograph. Nonetheless, our results should be verified with larger samples in future prospective studies. Second, fusion mass residual Cobb angle, which was not analyzed in this study, may have additional effects on the surgical outcomes that require further study. Similar to the methodology of this study in standardizing the fusion mass residual Cobb angle for analysis, the fusion mass shift should be standardized to isolate the effects of the fusion mass residual Cobb angle on global spinal balance and adding-on.

In our study, patients with a fusion mass shift of $20 \mathrm{~mm}$ or less were less likely to have adding-on develop by the 2-year followup than were patients with a fusion mass shift greater than $20 \mathrm{~mm}$. Moreover by using ROC analysis, setting the cutoff to $18 \mathrm{~mm}$ increased specificity of identifying patients at risk for adding-on. Cao et al. [1] reported that a residual shoulder imbalance is a substantial risk factor for adding-on. Using our concept of fusion mass shift, it can be concluded that the adding-on observed in their series is a direct result of the patient's compensatory mechanisms to rebalance the unleveled shoulders. This can be seen from the case example in their study where a fusion mass shift contributed to a higher right shoulder [1]. The fusion mass shape was unbalanced and therefore the patient can only rebalance with distal adding-on at L1. The culprit thus is inadequate squaring of the fusion mass and nonparallel upper and lower instrumented vertebral endplates. Cao et al. [1] also suggested that having too many unfused lumbar segments in a thoracic fusion allows more segments to accommodate for shoulder imbalance and thus a higher chance of having adding-on develop. Although fewer unfused segments with longer fusions may prevent adding-on, it will remove any compensatory mechanisms for fusion mass shift and result in fixed truncal shifting. Our observation is further justified considering that no statistical differences were observed between our two study groups regarding spinal balance and radiographic shoulder height. This limits the confounding effects of global balance on the association between fusion mass shift and adding-on.
The location of the lower instrumented vertebrae has been investigated in numerous studies $[6,34,52,53]$ as an important factor for adding-on. However, their analyses were flawed, as all lower instrumented vertebrae decisions were based on a single preoperative standing radiograph without consideration of curve flexibility. This was highlighted by Cho et al. [6], who suggested that the decision of fusion levels is dependent on the curve pattern but they did not explain why similar curves behave differently despite a similar fusion technique. They found that placing the lower instrumented vertebrae at the neutral vertebra is a high risk for adding-on and the stable and last-touched vertebra is the safer option with Lenke Types 1A-R AIS (L4 vertebra tilted to the right). In patients with the L4 vertebra tilting to the left (Lenke Types1A-L), fusing at least one level below the end vertebra is advised. Similar findings were observed by Wang et al. [52], who showed unsatisfactory outcomes with lower instrumented vertebrae at the end vertebra and lower instrumented vertebrae-center sacral vertical line distance greater than $10 \mathrm{~mm}$. The safe lower instrumented vertebrae was suggested as an additional vertebra caudal to the end vertebra or the first vertebra in the cephalad direction from the sacrum that deviates more than $10 \mathrm{~mm}$ from the center sacral vertical line [52, 53]. Matsumoto et al. [34] also recommended extending the lower instrumented vertebrae at least to the last vertebra touching the central sacral vertical line to avoid postoperative addingon. Although fusing an extra segment is safer, it violates the principle of fusing short or only what is necessary to achieve a balanced spine. In our study and the studies discussed here, there were patients who had satisfactory outcomes without adding-on despite fusing short. Therefore, curve flexibility is an important factor to consider as it may determine the behavior of the discs and vertebrae below the lower instrumented vertebrae. In addition, some examples of adding-on reported in the aforementioned studies had fusion mass shift which was not addressed. A third commonly discussed cause for adding-on is residual growth $[6,53]$. Residual lumbar curves may develop as a result of growth after surgery and it has been determined as an independent risk factor for adding-on [53]. However, we were unable to observe any substantial associations 
between skeletal immaturity and adding-on in our study, despite patients with Risser Stage 0 in both groups.

One of the main goals of AIS surgery is to reduce the number of fused segments while maintaining a balanced spine after surgery. Our concept of the fulcrum-bending radiograph is an important tool that helps us predict the behavior of a curve and determine fusion levels. The technique for using these radiographs to select the upper instrumented vertebrae and lower instrumented vertebrae were described in this study, and its utility in preventing fusion mass shift has been shown. Although the preoperative fulcrum-bending prediction may not always be achievable intraoperatively, it provides a predictive tool for the true curve flexibility and thus may indicate the minimal number of fusion segments that can achieve a squared fusion mass without shift. However, comparison between the fulcrum-bending radiograph and other imaging methods to reduce fusion mass shift requires further study.

We presume that the distal adding-on phenomenon may be a compensatory change for maintaining a well-balanced spine such as balanced shoulder level, truncal shift, and listing. We postulate that the shape of the fusion mass is highly associated with this compensatory mechanism. However, this requires further experimental work to prove. The clinical importance of adding-on is not fully understood, but extension of the primary curve can induce an increase of coronal decompensation and disc wedging, which can lead to degenerative changes later in life and/or the need for extending the fusion distally $[6,44]$. Therefore, and based on our ROC analysis, to prevent adding-on postoperatively, surgeons should pay attention to the measurements of fusion mass shift intraoperatively and keep it $20 \mathrm{~mm}$ or less.

\section{Conclusions}

The aim of AIS surgery is not only for Cobb angle correction, but for a balanced spine. This is necessary to achieve satisfactory outcomes and prevention of adding-on, which we believe is a direct consequence of fusion mass shift. Our criteria of accepting a 20-mm fusion mass shift and fusion mass Cobb angle of $20^{\circ}$ after surgery allows us to fuse as few lumbar segments as possible [30, 42]. Our study also underscores and verifies the importance of our criteria. Preoperative fulcrum-bending radiographs help surgeons select the fusion levels that predict a postoperative fusion mass shift $20 \mathrm{~mm}$ or less. Intraoperatively the fusion mass shift can be limited to our defined acceptable criteria by distraction or compression of the lower instrumented vertebrae. Squaring the fusion mass is key to not only achieving global balance but also in preventing adding-on. Our findings suggest that a residual fusion mass shift greater than $18 \mathrm{~mm}$ is strongly associated with distal adding-on after surgery. This phenomenon may represent a compensatory change to maintain a well-balanced spine owing to the residual fusion mass shift after surgery. Although fusion mass shift is not the only determining factor for adding-on, it is an important factor that should be considered and can be controlled by the surgeon intraoperatively during correction. Furthermore, the fusion mass shift is an important radiologic parameter for analysis of surgical outcomes that can be standardized between spine surgeons for comparative analyses.

\section{References}

1. Cao K, Watanabe K, Hosogane N, Toyama Y, Yonezawa I, Machida M, Yagi M, Kaneko S, Kawakami N, Tsuji T, Matsumoto M. Association of postoperative shoulder balance with adding-on in Lenke Type II adolescent idiopathic scoliosis. Spine (Phila Pa 1976). 2014;39:E705-712.

2. Cheung KM, Lam JW, Samartzis D, Lu WW, Luk KD. The use of a modified fulcrum for fulcrum bending radiographs: a technical note. J Orthop Surg (Hong Kong). 2014;22:248-251.

3. Cheung KM, Luk KD. Prediction of correction of scoliosis with use of the fulcrum bending radiograph. J Bone Joint Surg Am. 1997;79:1144-1150.

4. Cheung KM, Natarajan D, Samartzis D, Wong YW, Cheung WY, Luk KD. Predictability of the fulcrum bending radiograph in scoliosis correction with alternate-level pedicle screw fixation. $J$ Bone Joint Surg Am. 2010;92:169-176.

5. Cheung WY, Lenke LG, Luk KD. Prediction of scoliosis correction with thoracic segmental pedicle screw constructs using fulcrum bending radiographs. Spine (Phila Pa 1976). 2010;35:557-561.

6. Cho RH, Yaszay B, Bartley CE, Bastrom TP, Newton PO. Which Lenke 1A curves are at the greatest risk for adding-on.... and why? Spine (Phila Pa 1976). 2012;37:1384-1390.

7. Dobbs MB, Lenke LG, Kim YJ, Kamath G, Peelle MW, Bridwell $\mathrm{KH}$. Selective posterior thoracic fusions for adolescent idiopathic scoliosis: comparison of hooks versus pedicle screws. Spine (Phila Pa 1976). 2006;31:2400-2404.

8. Floman Y, Penny JN, Micheli LJ, Riseborough EJ, Hall JE. Osteotomy of the fusion mass in scoliosis. J Bone Joint Surg Am. 1982;64:1307-1316.

9. Fortin C, Feldman DE, Cheriet F, Labelle H. Validity of a quantitative clinical measurement tool of trunk posture in idiopathic scoliosis. Spine (Phila Pa 1976). 2010;35:E988-994.

10. Goldstein LA. The surgical management of scoliosis. Clin Orthop Relat Res. 1964;35:95-115.

11. Goldstein LA. The surgical management of scoliosis. Clin Orthop Relat Res. 1971;77:32-56.

12. Hamzaoglu A, Talu U, Tezer M, Mirzanli C, Domanic U, Goksan SB. Assessment of curve flexibility in adolescent idiopathic scoliosis. Spine (Phila Pa 1976). 2005;30:1637-1642.

13. Harrington PR. Treatment of scoliosis: correction and internal fixation by spine instrumentation. $J$ Bone Joint Surg Am. 1962;44:591-610.

14. Harrington PR. Technical details in relation to the successful use of instrumentation in scoliosis. Orthop Clin North Am. 1972;3:49-67.

15. Hay D, Izatt MT, Adam CJ, Labrom RD, Askin GN. The use of fulcrum bending radiographs in anterior thoracic scoliosis 
correction: a consecutive series of 90 patients. Spine (Phila Pa 1976). 2008;33:999-1005.

16. Kamerlink JR, Quirno M, Auerbach JD, Milby AH, Windsor L, Dean L, Dryer JW, Errico TJ, Lonner BS. Hospital cost analysis of adolescent idiopathic scoliosis correction surgery in 125 consecutive cases. J Bone Joint Surg Am. 2010;92:1097-1104.

17. Khuffash B, Porter RW. Cross leg pain and trunk list. Spine (Phila Pa 1976). 1989;14:602-603.

18. Kim YJ, Lenke LG, Cho SK, Bridwell KH, Sides B, Blanke K. Comparative analysis of pedicle screw versus hook instrumentation in posterior spinal fusion of adolescent idiopathic scoliosis. Spine (Phila Pa 1976). 2004;29:2040-2048.

19. King HA, Moe JH, Bradford DS, Winter RB. The selection of fusion levels in thoracic idiopathic scoliosis. J Bone Joint Surg Am. 1983;65:1302-1313.

20. Kleinman RG, Csongradi JJ, Rinksy LA, Bleck EE. The radiographic assessment of spinal flexibility in scoliosis: a study of the efficacy of the prone push film. Clin Orthop Relat Res. 1982;162:47-53.

21. Lenke LG, Betz RR, Bridwell KH, Harms J, Clements DH, Lowe TG. Spontaneous lumbar curve coronal correction after selective anterior or posterior thoracic fusion in adolescent idiopathic scoliosis. Spine. 1999;24:1663-1671; discussion 1672.

22. Lenke LG, Betz RR, Harms J, Bridwell KH, Clements DH, Lowe TG, Blanke K. Adolescent idiopathic scoliosis: a new classification to determine extent of spinal arthrodesis. J Bone Joint Surg Am. 2001;83:1169-1181.

23. Li J, Cheung KM, Samartzis D, Ganal-Antonio AK, Zhu X, Li M, Luk KD. Key -vertebral screws strategy for main thoracic curve correction in patients with adolescent idiopathic scoliosis. Clin Spine Surg. 2016;29:E434-441.

24. Li J, Dumonski ML, Samartzis D, Hong J, He S, Zhu X, Wang C, Vaccaro AR, Albert TJ, Li M. Coronal deformity correction in adolescent idiopathic scoliosis patients using the fulcrum-bending radiograph: a prospective comparative analysis of the proximal thoracic, main thoracic, and thoracolumbar/lumbar curves. Eur Spine J. 2011;20:105-111.

25. Little JP, Adam C. Towards determining soft tissue properties for modelling spine surgery: current progress and challenges. Med Biol Eng Comput. 2012;50:199-209.

26. Little JP, Adam CJ. The effect of soft tissue properties on spinal flexibility in scoliosis: biomechanical simulation of fulcrum bending. Spine (Phila Pa 1976). 2009;34:E76-82.

27. Lowenstein JE, Matsumoto H, Vitale MG, Weidenbaum M, Gomez JA, Lee FY, Hyman JE, Roye DP Jr. Coronal and sagittal plane correction in adolescent idiopathic scoliosis: a comparison between all pedicle screw versus hybrid thoracic hook lumbar screw constructs. Spine (Phila Pa 1976). 2007;32:448-452.

28. Luk KD, Cheung KM, Lu DS, Leong JC. Assessment of scoliosis correction in relation to flexibility using the fulcrum bending correction index. Spine (Phila Pa 1976). 1998;23:2303-2307.

29. Luk KD, Cheung WY, Wong Y, Cheung KM, Wong YW, Samartzis D. The predictive value of the fulcrum bending radiograph in spontaneous apical vertebral derotation in adolescent idiopathic scoliosis. Spine (Phila Pa 1976). 2012;37:E922926.

30. Luk KD, Don AS, Chong CS, Wong YW, Cheung KM. Selection of fusion levels in adolescent idiopathic scoliosis using fulcrum bending prediction: a prospective study. Spine (Phila Pa 1976). 2008;33:2192-2198.

31. Luk KD, Lee FB, Leong JC, Hsu LC. The effect on the lumbosacral spine of long spinal fusion for idiopathic scoliosis: a minimum 10-year follow-up. Spine (Phila Pa 1976). 1987;12:996-1000.

32. Luk KD, Vidyadhara S, Lu DS, Wong YW, Cheung WY, Cheung KM. Coupling between sagittal and frontal plane deformity correction in idiopathic thoracic scoliosis and its relationship with postoperative sagittal alignment. Spine (Phila $\mathrm{Pa}$ 1976). 2010;35:1158-1164.

33. Marks M, Newton PO, Petcharaporn M, Bastrom TP, Shah S, Betz R, Lonner B, Miyanji F. Postoperative segmental motion of the unfused spine distal to the fusion in 100 patients with adolescent idiopathic scoliosis. Spine (Phila Pa 1976). 2012;37:826832.

34. Matsumoto M, Watanabe K, Hosogane N, Kawakami N, Tsuji T, Uno K, Suzuki T, Ito M, Yanagida H, Yamaguchi T, Minami S, Akazawa T. Postoperative distal adding-on and related factors in Lenke type 1A curve. Spine (Phila Pa 1976). 2013;38:737-744.

35. McLean IP, Gillan MG, Ross JC, Aspden RM, Porter RW. A comparison of methods for measuring trunk list: a simple plumbline is the best. Spine (Phila Pa 1976). 1996;21:1667-1670.

36. Moe JH. A critical analysis of methods of fusion for scoliosis; an evaluation in two hundred and sixty-six patients. $J$ Bone Joint Surg Am. 1958;40:529-554 passim.

37. Nohara A Kawakami N, Seki K, Tsuji T, Ohara T, Saito T, Kawakami K. The effects of spinal fusion on lumbar disc degeneration in patients with adolescent idiopathic scoliosis: a minimum 10-year follow-up. Spine Deform. 2015;3:462-468.

38. Omidi-Kashani F, Hasankhani EG, Moradi A, Toossi KZ, Nojomi M. Modified fulcrum bending radiography: a new combined technique that may reflect scoliotic curve flexibility better than other conventional methods. J Orthop. 2013;10:172-176.

39. Parisini P, Di Silvestre M, Lolli F, Bakaloudis G. Selective thoracic surgery in the Lenke type 1A: King III and King IV type curves. Eur Spine J. 2009;18(suppl 1):82-88.

40. Porter RW, Miller CG. Back pain and trunk list. Spine (Phila Pa 1976). 1986;11:596-600.

41. Risser JC. Scoliosis: past and present. J Bone Joint Surg Am. 1964:46:167-199.

42. Samartzis D, Leung Y, Shigematsu H, Natarajan D, Stokes O, Mak KC, Yao G, Luk KD, Cheung KM. Selection of fusion levels using the fulcrum bending radiograph for the management of adolescent idiopathic scoliosis patients with alternate level pedicle screw strategy: clinical decision-making and outcomes. PloS One. 2015;10:e120302.

43. Smyrnis PN, Sekouris N, Papadopoulos G. Surgical assessment of the proximal thoracic curve in adolescent idiopathic scoliosis. Eur Spine J. 2009;18:522-530.

44. Sponseller PD, Betz R, Newton PO, Lenke LG, Lowe T, Crawford A, Sucato D, Lonner B, Marks M, Bastrom T. Differences in curve behavior after fusion in adolescent idiopathic scoliosis patients with open triradiate cartilages. Spine (Phila $\mathrm{Pa}$ 1976). 2009;34:827-831.

45. Suk SI, Lee CK, Min HJ, Cho KH, Oh JH. Comparison of CotrelDubousset pedicle screws and hooks in the treatment of idiopathic scoliosis. Int Orthop. 1994;18:341-346.

46. Suk SI, Lee SM, Chung ER, Kim JH, Kim SS. Selective thoracic fusion with segmental pedicle screw fixation in the treatment of thoracic idiopathic scoliosis: more than 5-year follow-up. Spine (Phila Pa 1976). 2005;30:1602-1609.

47. Sun YQ, Samartzis D, Cheung KM, Wong YW, Luk KD. The "X-Factor" index: a new parameter for the assessment of adolescent idiopathic scoliosis correction. Eur Spine J. 2011;20:144150 .

48. Thompson JP, Transfeldt EE, Bradford DS, Ogilvie JW, BoachieAdjei O. Decompensation after Cotrel-Dubousset instrumentation of idiopathic scoliosis. Spine (Phila Pa 1976). 1990;15:927-931.

49. Trobisch PD, Samdani AF, Pahys JM, Cahill PJ. Postoperative trunk shift in Lenke 1 and 2 curves: how common is it? and analysis of risk factors. Eur Spine J. 2011;20:1137-1140.

50. Vangeneugden T, Laenen A, Geys H, Renard D, Molenberghs G. Applying concepts of generalizability theory on clinical trial data 
to investigate sources of variation and their impact on reliability. Biometrics. 2005;61:295-304.

51. Vaughan JJ, Winter RB, Lonstein JE. Comparison of the use of supine bending and traction radiographs in the selection of the fusion area in adolescent idiopathic scoliosis. Spine (Phila Pa 1976). 1996;21:2469-2473.

52. Wang Y, Bunger CE, Zhang Y, Wu C, Li H, Hansen ES. Distal adding-on in Lenke 1A scoliosis: how to more effectively determine the onset of distal adding-on. Spine (Phila Pa 1976). 2013;38:490-495.

53. Wang Y, Hansen ES, Hoy K, Wu C, Bunger CE. Distal adding-on phenomenon in Lenke 1A scoliosis: risk factor identification and treatment strategy comparison. Spine (Phila Pa 1976). 2011;36:1113-1122.

54. Winter RB, Lonstein JE, Denis F. How much correction is enough? Spine (Phila Pa 1976). 2007;32:2641-2643. 